\title{
Papel de la coagulación en la fisiopatología de la lesión pulmonar aguda. Paralelismo con la sepsis
}

\author{
R. FERRER, A. NAVAS, M. ADDA Y A. ARTIGAS
}

Centro de Críticos. Hospital de Sabadell. Corporación Sanitaria Parc Taulí. CIBER Enfermedades Respiratorias. Instituto Universitario Parc Taulí. Universidad Autónoma. Barcelona. España.

La lesión pulmonar aguda y, en su forma más grave, el síndrome de distrés respiratorio agudo, tiene una alta morbimortalidad y se caracteriza por un inicio brusco, hipoxemia e infiltrados bilaterales en la radiografía de tórax con ausencia de signos de insuficiencia cardiaca. En la fisiopatología de la lesión pulmonar aguda participa, junto a la activación de la inflamación, una alteración del sistema de la coagulación.

Las alteraciones del sistema de la coagulación presentes en la lesión pulmonar aguda incluyen un aumento de la actividad procoagulante, así como un descenso de la actividad fibrinolítica, tanto en el espacio alveolar como en el intersticio pulmonar. Los depósitos de fibrina, que son el marcador inicial de la fase precoz de la lesión pulmonar aguda, estimulan la agregación de fibroblastos y la subsiguiente secreción de colágeno que conduce a la fibrosis pulmonar.

El único tratamiento que ha demostrado tener un impacto significativo sobre la mortalidad en la lesión pulmonar aguda/síndrome de distrés respiratorio agudo, a pesar de los avances sobre el conocimiento de la enfermedad en los últimos 10 años, es el uso de ventilación protectora con volumen corriente bajo. En la sepsis grave donde también participa la coagulación, sólo el tratamiento con proteína $\mathbf{C}$ activada ha mostrado una reducción de la mortalidad junto con una mejoría de la disfunción respiratoria y descenso en los días de ventilación mecánica.

Correspondencia: Dr. R. Ferrer.

UCI. Centro de Críticos.

Hospital de Sabadell.

Parc Taulí, s/n

08208 Sabadell. Barcelona. España.

Correo electrónico: rferrer@tauli.cat

Manuscrito aceptado el 3-IX-2007.
En el futuro, para determinar el papel de los anticoagulantes en el tratamiento de la lesión pulmonar aguda/síndrome de distrés respiratorio agudo, los próximos estudios deberían considerar el potencial beneficio de los anticoagulantes aplicados a nivel sistémico o local pulmonar.

PALABRAS CLAVE: síndrome de distrés respiratorio agudo, sepsis, drotrecogina alfa activada, fibrina.

\section{ROLE OF COAGULATION IN ACUTE PULMONARY LESION PHYSIOPATHOLOGY. PARALLELISM WITH SEPSIS}

Acute respiratory distress syndrome and acute lung injury for a part of a devastating syndrome characterized by acute onset, hypoxemia and bilateral infiltrates in the chest $\mathrm{x}$-ray with absence of heart failure signs. Acute lung injury is the response of the lung to a local or systemic aggression, resulting in local inflammation and coagulation disorders, this leading to increased inflammatory pulmonary edema.

Acute lung injury/acute respiratory distress syndrome are associated with increased procoagulant and reduced fibrinolytic activities mainly in alveoli and interstitial spaces in the lung. Fibrin deposits, which are the hallmark of early phase acute lung injury, stimulate fibroblast aggregation and collagen secretion, participating to the constitution of pulmonary fibrosis.

The only clinical intervention found to have a significant impact on mortality in acute respiratory distress syndrome, despite the significant progress in the understanding of the disease made over the past 10 years, is the use of low tidal volume ventilation. In severe sepsis, only recombinant human activated protein $\mathbf{C}$ administration 
has demonstrated a mortality reduction, together with faster improvement in respiratory dysfunction and shorter duration of mechanical ventilation.

Future clinical trials should consider the potential benefit of anticoagulants administrated systemically or locally in the lungs to determine the role of anticoagulants in the treatment of acute pulmonary injury/acute respiratory distress syndrome.

KEY WORDS: acute respiratory distress syndrome, sepsis, drotrecogin alfa (activated), fibrin.

\section{INTRODUCCIÓN}

El síndrome de distrés respiratorio agudo (SDRA) se caracteriza por un inicio brusco, hipoxemia (relación $\mathrm{PaO}_{2} / \mathrm{FiO}_{2} \leq 200 \mathrm{mmHg}$ ) e infiltrados bilaterales en la radiografía de tórax en ausencia de signos clínicos de insuficiencia cardiaca. El SDRA es una de las causas de mayor morbimortalidad en las Unidades de Cuidados Intensivos, y se asocia a unos costes muy elevados. Los últimos estudios reportan una mortalidad de un 40-60\%. El diagnóstico de SDRA y su forma menos grave, la lesión pulmonar aguda (LPA), definida por una relación $\mathrm{PaO}_{2} / \mathrm{FiO}_{2} \leq 300 \mathrm{mmHg}$ es clínico ${ }^{1}$. El riesgo para el desarrollo de SDRA/LPA depende de la presencia de los potenciales agentes causales y de la sensibilidad individual. Las principales etiologías causantes de este síndrome incluyen la sepsis, la neumonía, la broncoaspiración, el traumatismo, la pancreatitis, las politransfusiones, la inhalación de humo o gases tóxicos y diversas formas de toxicidad por fármacos ${ }^{2}$. La presencia de múltiples factores predisponentes, como la enfermedad pulmonar crónica o la acidosis metabólica, se asocian con una mayor probabilidad de desarrollar SDRA/LPA. A menudo se combinan diferentes factores etiológicos, lo que aumenta la probabilidad de desarrollo de este síndrome.

El SDRA/LPA se produce como respuesta del pulmón a una agresión local o sistémica, generando inflamación local y trastornos de la coagulación que provocarán un aumento del edema pulmonar inflamatorio $^{3}$. Los estudios anatomopatológicos de pacientes fallecidos por SDRA/LPA muestran daño alveolar difuso con un aumento del número de neutrófilos, plaquetas y fibrina, tanto a nivel intravascular como extravascular, así como lesión del endotelio y el epitelio alveolar ${ }^{4}$

La sepsis grave se define como la presencia de una infección sospechada o probada junto con síndrome de respuesta inflamatoria sistémica y una o más disfunciones orgánicas (tensión arterial sistólica [TAS] $<90 \mathrm{mmHg}$, hipoxemia, oliguria, acidosis metabólica o trombocitopenia $)^{5}$. La disfunción respiratoria (incluyendo SDRA/LPA) es frecuente en la sepsis, y los pacientes habitualmente precisan ventilación mecánica para evitar la fatiga muscular, garantizar el intercambio gaseoso y proteger la vía aérea de la broncoaspiración. Los pacientes intubados y conectados a ventilación mecánica son más propensos al desarrollo de una neumonía, la cual es causante de una morbilidad y mortalidad adicional ${ }^{6}$. Además, la ventilación mecánica por sí misma puede agravar o incluso iniciar la inflamación pulmonar, empeorando aún más el pronóstico ${ }^{7}$. De hecho, el único tratamiento que ha demostrado tener un impacto significativo sobre la mortalidad en el SDRA, a pesar de los avances sobre el conocimiento de la enfermedad en los últimos 10 años, es el uso de ventilación protectora ${ }^{8}$.

El SDRA/LPA y la sepsis comparten diversas similitudes, como la presencia de fallo multiorgánico y el estado de hipercoagulabilidad con la formación de microtrombos vasculares ${ }^{9}$. Uno de los pilares del tratamiento de la sepsis es devolver el equilibrio a la hemostasia, tal como quedó demostrado con el uso de proteína $\mathrm{C}$ activada (PCA) ${ }^{10}$. Dado que en los pacientes con SDRA/LPA se han encontrado importantes alteraciones de la coagulación y la fibrinólisis muy similares a las encontradas en aquellos con sepsis gra$\mathrm{ve}^{11}$, los anticoagulantes usados en el tratamiento de la sepsis podrían mejorar la evolución clínica de los pacientes con SDRA.

\section{LA COAGULACIÓN Y LA INFLAMACIÓN EN LA SEPSIS}

\section{Trastornos de la hemostasia en la sepsis}

La respuesta inicial contra la infección es neurohumoral, activando la respuesta pro- y antiinflamatoria. Se inicia con la activación celular de monocitos, macrófagos y neutrófilos que interactúan con las células endoteliales a través de múltiples receptores de reconocimiento de patógenos. La respuesta del huésped, tras la activación celular, incluye la movilización de sustancias plasmáticas (citoquinas, factor de necrosis tumoral [TNF], interleucinas [IL], caspasa, proteasas, leucotrienos, quininas, óxido nítrico, ácido araquidónico, factor activador de plaquetas y eicosanoides). La activación del complemento y la coagulación actúan amplificando la cadena de sucesos.

La activación de la coagulación en la sepsis se produce a través de la vía extrínseca de la coagulación. El factor tisular (FT) junto con el factor VII activado (FVIIa) forman el complejo FT-FVIIa, que cataliza la conversión de factor X (FX) a FX activado (FXa), el cual junto con el factor $\mathrm{V}$ activado (FVa), la protrombina y el calcio generan el complejo FXa-Va que forma la trombina (FIIa), que finalmente genera fibrina a través del fibrinógeno.

En circunstancias normales sólo pequeñas cantidades de FT están expuestas en la circulación sanguínea, pero en estados de gran inflamación, tanto células endoteliales como monocitos expresan FT en su superficie. También algunas citoquinas proinflamatorias, como el TNF $\alpha$ y las IL 1 y 6 , pueden aumentar la expresión de FT. La expresión in vivo del FT 
parece depender mayoritariamente de la IL-6. El aumento de la actividad coagulante no está suficientemente contrabalanceada por los inhibidores naturales de la coagulación durante la sepsis grave.

Los mecanismos que regulan la coagulación, en situaciones normales, incluyen los inhibidores naturales de la coagulación, tales como la PCA, antitrombina III (AT) y el inhibidor de la vía del factor tisular (TFPI). Todos ellos interfieren a diferente nivel con la activación de la coagulación por el complejo FT-FVIIa. La PCA inactiva los factores Va y VIIIa, la AT neutraliza varias enzimas en la cascada de la coagulación, incluyendo la trombina, y el TFPI interfiere con el factor Xa e inhibe el complejo FT-FVIIa. En los pacientes con sepsis los niveles de AT y de proteína C están disminuidos, resultado de un alto consumo y degradación y una inadecuada síntesis ${ }^{12}$. Los niveles basales de proteína $\mathrm{C}$ y el descenso de los niveles después del primer día de evolución de la sepsis también son un predictor independiente de mortalidad ${ }^{13}$.

Por otro lado, existe un déficit de activación de la proteína $\mathrm{C}$ debido a que la trombomodulina, principal mediador de la activación de la proteína $\mathrm{C}$ (proceso dependiente de trombina) se encuentra inhibida en la superficie endotelial por las citoquinas proinflamatorias endoteliales como el TNF $\alpha$ y la IL-1, generando una disfunción del sistema de la proteína $\mathrm{C}^{14}$. La importancia de esta proteína ha sido demostrada repetidamente en numerosos estudios preclínicos y en un ensayo clínico, constatando que la infusión de PCA recombinante mejora la supervivencia en los pacientes con sepsis grave ${ }^{10}$.

En cuanto a la fibrinólisis, la respuesta inicial ante una infección es la activación, mediada por los activadores de plasminógeno del endotelio. Esta respuesta es de corta duración, dando paso a una rápida síntesis del inhibidor del activador del plasminógeno (PAI-1). El PAI-1 es el principal inhibidor del activador tisular del plasminógeno (tPA) y del activador del plasminógeno tipo uroquinasa (uPA). Tanto el TNF $\alpha$ como la IL-1 han mostrado tener efectos antifibrinolíticos, bien por estimulación de la liberación de PAI-1, bien reduciendo la liberación de $\mathrm{tPA}^{15}$ (fig. 1).

\section{Efecto amplificador recíproco de la inflamación y la coagulación}

Existen múltiples conexiones entre los mediadores inflamatorios y los productos de la coagulación, generándose una modulación recíproca. El principal iniciador de la formación de trombina es el FT, dependiente principalmente de la expresión de IL-6. La activación de la coagulación y los depósitos de fibrina son consecuencia de las proteasas que interactúan con receptores específicos celulares que inducen señales que mediarán la respuesta inflamatoria, transformando productos de la coagulación en señales inflamatorias ${ }^{16}$. El principal mecanismo por el que estas proteasas actúan sobre la inflamación es a través de receptores activados por proteasas (PAR), de los cuales se han identificado 4 tipos (PAR 1-4). Estas pro-

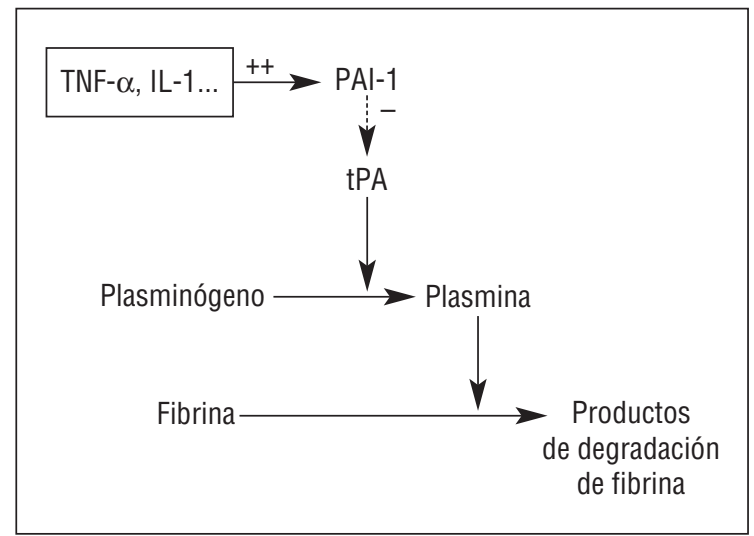

Figura 1. Inhibición del sistema de la fibrinólisis. Mecanismo de acción del inhibidor del activador del plasminógeno (PAI-1). IL: interleucina; TNF: factor de necrosis tumoral; tPA: activador tisular del plasminógeno.

teínas transmembrana se expresan en la superficie de las células endoteliales, los monocitos, plaquetas, fibroblastos y células lisas musculares. Los PAR 1, 3 y 4 son receptores de trombina, y el PAR-2 puede ser activado por el complejo FT-FVIIa. Su activación tiene un fuerte efecto proinflamatorio, con generación adicional de citoquinas proinflamatorias y aumento de expresión de proteínas de superficie que incrementarán la extravasación de leucocitos.

\section{COAGULACIÓN E INFLAMACIÓN EN LA LESIÓN PULMONAR AGUDA}

El SDRA/LPA y la sepsis comparten diversas similitudes, como la presencia de fallo multiorgánico y el estado de hipercoagulabilidad con la formación de microtrombos vasculares ${ }^{9}$.

La existencia de actividad procoagulante en el SDRA/LPA es conocida desde hace 30 años. En 1976 Bone et $\mathrm{al}^{17}$ publicaron sus hallazgos autópsicos en pacientes fallecidos por SDRA. Observaron microtrombos de fibrina en los pulmones, tanto en presencia como en ausencia de coagulación intravascular diseminada (CID). Además, se observó que la reducción de la compliancia pulmonar y el empeoramiento del intercambio gaseoso se correlacionaban con la presencia de CID.

Las características morfológicas del SDRA/LPA incluyen los depósitos intraalveolares e intersticiales de fibrina. La LPA se caracteriza por un aumento de la actividad procoagulante ${ }^{18}$, así como por un descenso de la actividad fibrinolítica en el alveolo pulmonar, lo cual genera los depósitos de fibrina, presentes ya en las fases más precoces del daño pulmonar ${ }^{11}$.

De forma similar a la sepsis, la activación de la coagulación en la LPA es a través de la vía extrínseca de la coagulación ${ }^{19}$. Los niveles de FT están aumentados en el lavado broncoalveolar (LBA) de estos pacientes, desempeñando un papel importante en el estado de hipercoagulabilidad asociado al SDRA. Esta actividad procoagulante está aumentada, de forma signi- 
TABLA 1. Modulación de la coagulación y la fibrinólisis en lavado broncoalveolar de pacientes con lesión pulmonar aguda y síndrome de distrés respiratorio agudo

\begin{tabular}{|c|c|c|}
\hline & SDRA/LPA & Referencias \\
\hline Actividad procoagulante & $\Uparrow$ & \\
\hline Factor tisular soluble & $\uparrow \uparrow$ & $18,19,21,22$ \\
\hline Factor VIIa & $\uparrow$ & 19,22 \\
\hline Anticoagulantes naturales & $\Downarrow$ & \\
\hline Proteína C & $\downarrow$ & 23,24 \\
\hline Antitrombina & $\downarrow$ & 19 \\
\hline Trombomodulina soluble & $\uparrow$ & 23 \\
\hline Actividad fibrinolítica & $\Downarrow$ & \\
\hline $\begin{array}{l}\text { Activador del plasminógeno tipo } \\
\text { uroguinasa }\end{array}$ & $\downarrow$ & 20 \\
\hline Activador tisular del plasminógeno & $\downarrow$ & 20 \\
\hline Inhibición de la fibrinólisis & $\Uparrow$ & \\
\hline Inhibidor del activador & & \\
\hline del plasminógeno-1 & $\uparrow \uparrow$ & 19,22 \\
\hline
\end{tabular}

$\Uparrow$ y $\uparrow:$ aumento; $\uparrow \uparrow:$ aumento importante; $\Downarrow$ y $\downarrow$ : descenso; LPA: lesión pulmonar aguda; SDRA: síndrome de distrés respiratorio agudo.

ficativa, durante el periodo precoz exudativo del SDRA y tiende a disminuir a los 7-14 días del inicio de los síntomas ${ }^{20}$. La actividad procoagulante es independiente del inicio del SDRA y se ha observado tanto en la lesión pulmonar de causa extrapulmonar como intrapulmonar, tales como una peritonitis secundaria $^{21}$ o una neumonía grave que requiera ventilación mecánica ${ }^{22}$.

Los niveles de anticoagulantes naturales como la proteína $\mathrm{C}$ o la trombomodulina también están disminuidos en sangre y en el LBA de pacientes con $\mathrm{SDRA}^{23}$, y los niveles plasmáticos de proteína $\mathrm{C}$ se correlacionan con el pronóstico ${ }^{24}$.
Nuevamente, y de forma similar a la sepsis, la actividad fibrinolítica está inhibida en la LPA. La actividad fibrinolítica está reducida o es indetectable en el LBA durante la fase exudativa precoz, y permanece baja hasta el día 14 de inicio del SDRA ${ }^{20}$, contribuyendo a la formación de fibrina en la lesión pulmonar. La actividad de la uroquinasa, en el LBA de pacientes con SDRA, está drásticamente reducida comparada con pacientes sanos, sin embargo PAI-1, PAI-2 y $\alpha 2$-antiplasmina están aumentados ${ }^{22,25}$. Los cambios en la coagulación y la fibrinólisis en el LBA se muestran en la tabla 1.

Además, la ventilación mecánica por sí misma puede inducir un estado procoagulante a nivel alveolar en individuos sin lesión pulmonar previa, que puede ser prevenida mediante el uso de «ventilación protectora» con volúmenes corrientes bajo y presión positiva al final de la espiración (PEEP) ${ }^{26}$. En pacientes con SDRA/LPA la aplicación de ventilación mecánica «no protectora» podría provocar el empeoramiento o la perpetuación del estado procoagulante alveolar.

Los depósitos de fibrina aumentan la respuesta inflamatoria a través del aumento de la permeabilidad vascular, que generará una activación de las células endoteliales que producen citoquinas proinflamatorias aumentando la permeabilidad vascular. También generan óxido nítrico, potente vasodilatador, que induce la acumulación de neutrófilos activados y modula la respuesta inflamatoria en el pulmón. La fibrina también es un gran inhibidor del surfactante pulmonar. Finalmente, los productos de degradación de la fibrina son un potente quimiotáctico para el reclutamiento de neutrófilos y son capaces de estimular la proliferación de fibroblastos ${ }^{27}$. Los fibroblastos se agregan y secretan colágeno induciendo la formación final de la fibrosis $\operatorname{pulmonar}^{28}$ (fig. 2).

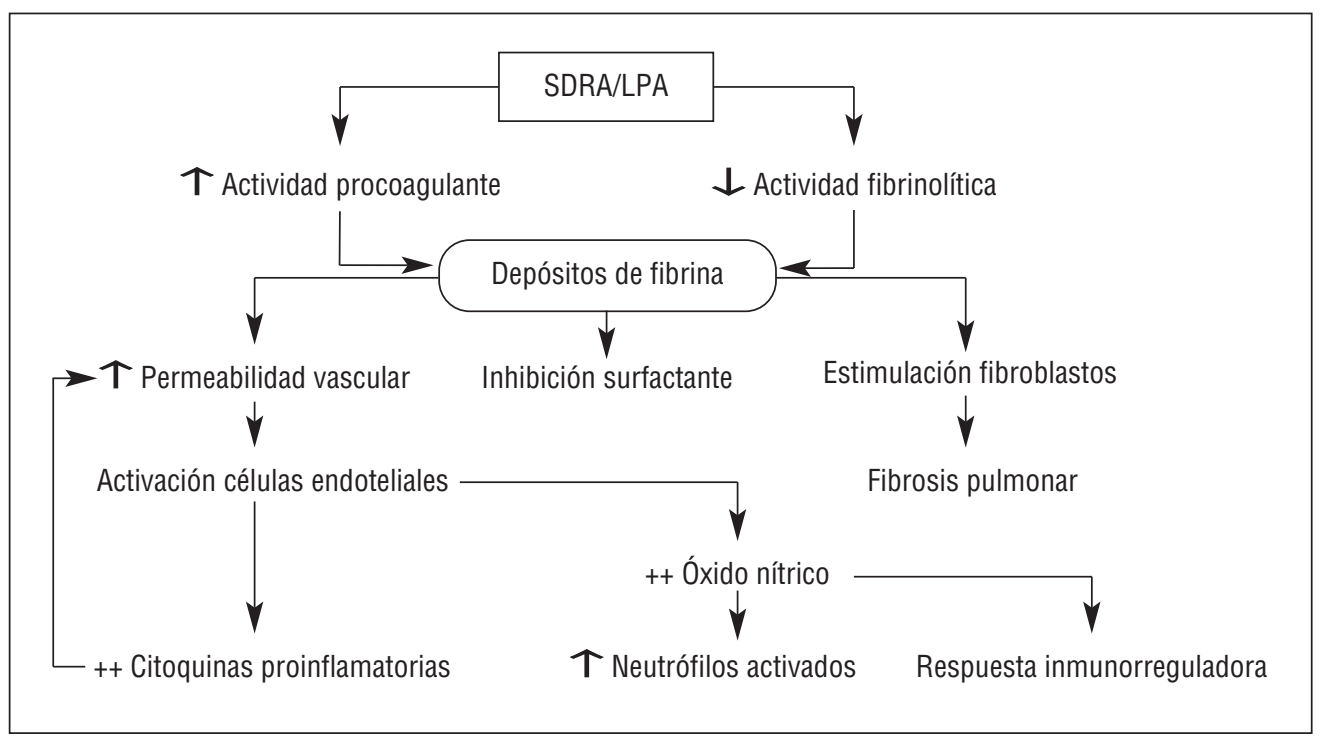

Figura 2. Consecuencias de la acumulación pulmonar de fibrina. LPA: lesión pulmonar aguda; SDRA: síndrome de distrés respiratorio agudo. 
En el SDRA/LPA un complejo de citoquinas y otras sustancias proinflamatorias inician y amplifican la respuesta inflamatoria en el pulmón, balanceada con la producción de mediadores antiinflamatorios ${ }^{29}$. La medición simultánea de los niveles plasmáticos de estas citoquinas proinflamatorias demuestra niveles bajos en comparación con los pulmonares, lo que evidencia que estos mediadores se originan predominantemente en el compartimiento pulmonar ${ }^{30,31}$.

Las múltiples conexiones existentes entre los sistemas de inflamación y coagulación desempeñan papeles muy importantes en el desarrollo del SDRA/ LPA. La activación de la coagulación y el depósito de fibrina secundarios a la inflamación pueden verse como una parte esencial de la defensa del huésped en el esfuerzo por contener al agente patógeno y confinar la inflamación al área pulmonar. Sin embargo, una respuesta incontrolada acaba convirtiéndose en un empeoramiento de la enfermedad.

Las alteraciones de los depósitos de fibrina no se limitan al espacio alveolar y/o intersticial, pudiendo encontrar trombos a nivel de las arterias pulmonares $^{32}$. En el estudio autópsico de pacientes con LPA se han evidenciado trombos en la arteria pulmonar, así como una disminución del grosor de la pared vascular. In vivo, mediante angiografía, se ha demostrado trombosis arterial y alteraciones distales en la microvasculatura pulmonar. Ambas se asocian con disfunción pulmonar, incluyendo atelectasias y un incremento de las resistencias vasculares pulmonares, así como un aumento de la mortalidad ${ }^{33}$. Los trombos en la arteria pulmonar pueden asociarse con un incremento del espacio muerto, que es considerado como un factor de mal pronóstico en el SDRA ${ }^{34}$. Por lo tanto, el depósito de fibrina tanto a nivel intravascular como extravascular parece contribuir al desarrollo de LPA, aunque todavía desconocemos cómo estos factores modulan el desarrollo del SDRA.

\section{Relación entre la fibrina, la inflamación alveolar y la fibrosis pulmonar}

Los depósitos de fibrina a nivel intravascular y extravascular son característicos de la LPA ${ }^{3}$. Varios estudios demuestran que la formación de los depósitos de fibrina es una transición hacia la fibrosis pulmonar ${ }^{35}$. En los inicios de la LPA los depósitos de fibrina pueden ejercer efectos beneficiosos sobre el intercambio gaseoso, al sellar los pequeños daños a nivel del endotelio y del epitelio. Por otro lado, no se han objetivado depósitos de fibrina, intravascular ni extravascular en el pulmón sano, por lo que su aparición en el SDRA muestra su importante causalidad. La fibrina ejerce influencia sobre la inflamación alveolar local y la reparación del tejido de diversas maneras: a) alterando la migración de células inflamatorias (macrófagos y fibroblastos); b) previniendo la proliferación linfocítica; c) evitando la organización de células endoteliales, y d) aumentando la permeabilidad vascular ${ }^{36}$.

El aumento de la expresión del FT está estimulado por diversas citoquinas, incrementando la formación de fibrina en el tejido dañado ${ }^{37}$. Las fibrinolisinas y el
PAI-1 interactúan con las citoquinas regulando el proceso de reparación tisular. El depósito temporal de fibrina en el espacio alveolar puede acelerar la reparación fibrótica ${ }^{38}$, lo que resultará en la remodelación de la pared intersticial alveolar. Esto comportará empeoramiento del intercambio gaseoso, así como de la compliancia pulmonar, y prolongará la dependencia de la ventilación mecánica. La función del surfactante puede alterarse por la fibrina o los productos de su degradación, potenciando las microatelectasias en el pulmón dañado ${ }^{39}$.

La amplificación de la respuesta inflamatoria puede ocurrir a través de la interacción de los mediadores de la coagulación y la fibrinólisis con componentes de otras vías inflamatorias, como el complemento o el sistema de las quininas. La expresión de los mediadores inflamatorios puede estar influenciada por componentes de la cascada de coagulación: la inducción de IL-1 puede estar regulada por el fibrinógeno y la trombina puede influenciar la expresión de citoquinas y aumentar la permeabilidad vascular a través de señales celulares ${ }^{40}$.

La trombina regula la contracción y permeabilidad de las células endoteliales, y participa en la quimiotaxis y regulación del tráfico de células inflamatorias. Ha sido recientemente demostrado que la trombina es capaz de inducir daño sobre las células epiteliales alveolares provocando un aumento de la permeabilidad ${ }^{41}$.

Las células que residen en el pulmón -incluyendo células epiteliales, fibroblastos y macrófagos alveolares- expresan FT, uPA, tPA y PAI 1 y 2 , influenciando de ese modo la actividad fibrinolítica en el compartimiento alveolar. El FT es expresado por las células pulmonares ${ }^{9,42,43}$, sin embargo la expresión del FT por fibroblastos y células endoteliales y epiteliales es inducible por daño tisular o por estímulos inflamatorios tales como la IL-6 o el TNF $\alpha$. Por tanto, varias células pulmonares podrían contribuir al aumento de la actividad procoagulante que se encuentra implicada en los procesos de lesión y reparación celular.

En el SDRA, en los pacientes a los que se les ha realizado LBA tras tres días de diagnóstico del síndrome, se han objetivado grandes incrementos de la actividad procoagulante sumado a grandes descensos de la actividad fibrinolítica. La progresión del SDRA a las dos semanas muestra un descenso de la actividad procoagulante alveolar gradual, mientras que la actividad fibrinolítica permanece todavía muy baja ${ }^{20}$. Todo ello contribuye a la persistencia de fibrina en el alveolo, que finalizará con el remodelado y fibrosis pulmonar. Las mayores concentraciones de PAI-1 encontradas en plasma de pacientes con SDRA frente a pacientes graves de control ${ }^{44}$ sugiere que alteraciones locales de la fibrinólisis en el SDRA no están compartimentadas y podrían tener repercusión sistémica.

\section{COAGULOPATÍA PULMONAR COMO OBJETIVO TERAPÉUTICO}

Dadas las similitudes entre la sepsis y la inflamación pulmonar en relación con la coagulopatía, y que la mayor causa de muerte en el SDRA/LPA no es el 


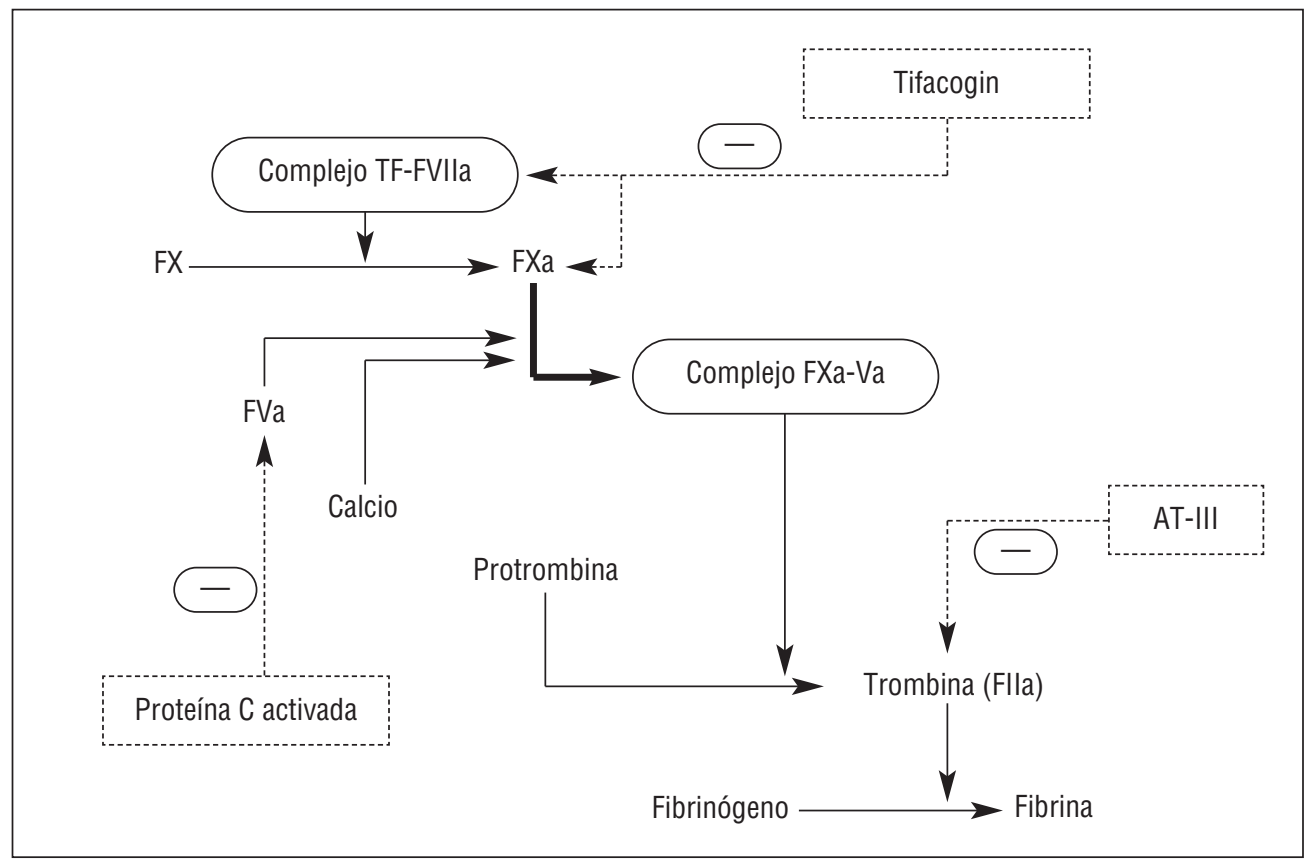

Figura 3. Mecanismo de acción de los inhibidores de la coagulación evaluados para el tratamiento de la sepsis grave. AT-III: antitrombina-III; FT: factor tisular.

fallo respiratorio, sino el fallo multiorgánico, se ha especulado que el tratamiento anticoagulante o profibrinolítico podría ser beneficioso en el SDRA/LPA ${ }^{45}$. La PCA recombinante demostró una reducción significativa de la mortalidad en los pacientes con sepsis grave $^{10}$. Otros fármacos, como la antitrombina III y el TFPI, que interfieren en la vía procoagulante, han sido motivo de estudios aleatorizados, pero no demostraron reducciones significativas en la mortalidad de pacientes con sepsis grave ${ }^{46,47}$. Una de las explicaciones podría ser que la actividad anticoagulante, per se, no es suficiente para la eficacia del tratamiento. A pesar de todo, los hallazgos fisiopatológicos en los pulmones sugieren que la teoría de bloquear el inicio de la coagulación podría cambiar el curso de la LPA y el SDRA $^{11}$. El mecanismo de acción de los anticoagulantes se muestra en la figura 3 .

\section{Proteína C}

La vía de la proteína $\mathrm{C}$ desempeña un importante papel en la regulación de la formación de trombina. La proteína $\mathrm{C}$, cuando se activa por el complejo trombina-trombomodulina, inactiva los factores $\mathrm{Va} y$ VIIIa, y finalmente impide la generación de trombina. La actividad antitrombótica de la PCA se asocia con acciones profibrinolíticas. La PCA inhibe la actividad de PAI-1 in vitro y puede, disminuyendo la formación de trombina, limitar la activación del inhibidor de la fibrinólisis activable por trombina ${ }^{48}$.

Se ha objetivado un papel antiinflamatorio de la PCA in vitro. Usando concentraciones por encima de las farmacológicas se mostró un descenso en la ex- presión de citoquinas inflamatorias como la IL-1 o TNF $\alpha$. Sin embargo, no se ha podido reproducir en estudios clínicos, en plasma ni en $\mathrm{LBA}^{49}$. De forma más reciente se ha demostrado que la PCA también modula la función endotelial mediante la interacción con el receptor de la proteína $\mathrm{C}$ endotelial (EPCR) y el PAR-1 a través de la vía de la esfingosina-1-fosfato y de la vía Rho-quinasa. La PCA a través de los EPCR también modula la quimiotaxis de los leucoci$\operatorname{tos}^{50}$.

En el estudio PROWESS se analizó la eficacia de la administración de PCA humana recombinante en pacientes con sepsis grave, demostrando una reducción significativa de la mortalidad ${ }^{10}$. Algunos pacientes incluidos en el estudio tenían concomitantemente SDRA y algunos padecían neumonía como causa primaria de la sepsis. En este estudio la administración de PCA se asoció con una resolución más rápida de la disfunción respiratoria y un aumento de los días de libre ventilación, comparado con placebo $(14,3$ frente a 13,2 días respectivamente; $p=0,049)^{51}$. El hecho de que estos resultados no se hayan podido reproducir en el subgrupo de pacientes con menor gravedad (estudio ADDRESS) ${ }^{52}$ ha generado controversia ${ }^{53}$ y ha limitado la indicación de PCA a pacientes con sepsis grave y alto riesgo de muerte, siendo el SDRA una de las situaciones que se asocian a mayor mortalidad.

De hecho, la PCA ejerce un efecto anticoagulante en el pulmón humano sometido a endotoxina y paralelamente reduce la actividad del PAI- ${ }^{54}$. Por lo tanto, la administración sistémica de PCA podría influenciar el depósito de fibrina en el pulmón. Actualmente está en marcha un ensayo clínico doble cie- 
go, aleatorizado, fase II, para evaluar el uso de PCA en el tratamiento de la LPA.

\section{Inhibidor del factor tisular}

La administración de TFPI recombinante (tifacogin) ha sido evaluada en un estudio fase II en pacientes con sepsis grave. Aunque no se mostró un beneficio estadísticamente significativo sobre la mortalidad, sí se objetivó una reducción relativa de mortalidad a los 28 días en un $20 \%$ en los pacientes tratados con tifacogin, junto con una mejoría en la disfunción orgánica. De forma interesante se produjo una reducción de la mortalidad en los pacientes con sepsis grave y SDRA que fueron tratados con tifacogin (37\% frente a 57\%) y la función pulmonar mejoró. Sin embargo, la eficacia del tifacogin en pacientes con sepsis grave no se confirmó en un estudio fase III (OPTIMIST) ${ }^{46}$. Un análisis de un subgrupo de este estudio sugiere que el tifacogin puede ser efectivo en pacientes con neumonía grave adquirida en la comunidad no tratados con heparina. En un modelo animal de SDRA inducido por sepsis se demostró que el pretratamiento con TFPI protege del desarrollo de disfunción pulmonar y del depósito de fibrina extravascular pulmonar ${ }^{55}$.

Actualmente está en curso un estudio fase III, internacional, controlado con placebo (CAPTIVATE), que evalúa el tratamiento en pacientes con neumonía grave adquirida en la comunidad.

\section{Otros anticoagulantes}

La administración de altas dosis de antitrombina III no modificó la mortalidad en la sepsis ${ }^{47}$. Mejorías en la lesión pulmonar, observadas en animales tratados con AT, no se han confirmado en humanos ${ }^{45}$. Hasta la fecha no se han realizado estudios sobre el efecto de los inhibidores naturales de la coagulación sobre pacientes con SDRA/LPA sin sepsis; sin embargo el tratamiento con tPA o uPA desciende la mortalidad y proporciona protección pulmonar en un modelo porcino de SDRA $^{56}$. El tratamiento con uPA mejoraba la hemodinámica y la oxigenación, así como angiográficamente se objetivaba la desaparición de los trombos a nivel pulmonar en los pacientes con LPA $^{57}$. Las evidencias sugieren que el uso de fármacos fibrinolíticos mejora la disfunción pulmonar en la LPA $^{35}$. La administración en aerosol de heparina o uroquinasa ha mostrado un efecto curativo en la fibrosis inducida por bleomicina en conejos. La anticoagulación o la fibrinólisis compartimentada, mediante la administración inhalada en el espacio alveolar, pueden ofrecer nuevas estrategias terapéuticas para la prevención de la fibrosis ${ }^{58}$.

\section{CONCLUSIONES}

La LPA y el SDRA se asocian con un aumento de la actividad procoagulante y una reducción de la actividad fibrinolítica, principalmente en el alveolo y en el espacio intersticial del pulmón, conduciendo a la formación de fibrina, que es el sello de la fase precoz de la LPA. Los depósitos de fibrina estimulan la agregación de los fibroblastos y la secreción de colágeno constituyendo la fibrosis pulmonar.

Únicamente la PCA humana recombinante, en pacientes con sepsis grave, ha mostrado una reducción significativa de la mortalidad, junto con una rápida mejoría de la disfunción respiratoria y una menor duración de la ventilación mecánica. Los futuros estudios clínicos en SDRA/LPA deberían evaluar el potencial beneficio de los anticoagulantes administrados bien sistémicamente, bien a nivel pulmonar.

\section{Declaración de conflicto de intereses}

Los Dres. Ferrer y Artigas han participado como ponentes en Reuniones Científicas organizadas y financiadas por Lilly.

El resto de los autores ha declarado no tener ningún conflicto de intereses.

\section{BIBLIOGRAFÍA}

1. Bernard GR, Artigas A, Brigham KL, Carlet J, Falke K, Hudson L, et al. The American-European Consensus Conference on ARDS. Definitions, mechanisms, relevant outcomes, and clinical trial coordination. Am J Respir Crit Care Med. 1994;149:818-24.

2. Hudson L, Milberg JA, Anardi D, Maunder RJ. Clinical risks for development of the acute respiratory distress syndrome. Am J Respir Crit Care Med. 1995;151:293-301.

3. Bachofen M, Weibel ER. Structural alterations of lung parenchyma in the adult respiratory distress syndrome. Clin Chest Med. 1982;3:35-56.

4. Ware LB, Matthay MA. The acute respiratory distress syndrome. N Engl J Med. 2000;342:1334-49.

5. Russell JA. Management of sepsis. N Engl J Med. 2006;355: 1699-713.

6. Ferrer R, Bauer T, Torres T. Nosocomial pneumonia during acute respiratory distress syndrome. Clin Intensive Care. 2001;12: 43-51.

7. Tremblay LN, Slutsky AS. Ventilator-induced lung injury: from the bench to the bedside. Intensive Care Med. 2006;32:24-33.

8. Ventilation with lower tidal volumes as compared with traditional tidal volumes for acute lung injury and the acute respiratory distress syndrome. The Acute Respiratory Distress Syndrome Network. N Engl J Med. 2000;342:1301-8.

9. Abraham E. Coagulation abnormalities in acute lung injury and sepsis. Am J Respir Cell Mol Biol. 2000;22:401-4.

10. Bernard GR, Vincent JL, Laterre PF, LaRosa SP, Dhainaut JF, Lopez-Rodriguez A, et al. Efficacy and safety of recombinant human activated protein C for severe sepsis. N Engl J Med. 2001; 344:699-709.

11. Schultz MJ, Haitsma JJ, Zhang H, Slutsky AS. Pulmonary coagulopathy as a new target in therapeutic studies of acute lung injury or pneumonia--a review. Crit Care Med. 2006;34:871-7.

12. Fourrier F, Chopin C, Goudemand J, Hendrycx S, Caron C, Rime A, et al. Septic shock, multiple organ failure, and disseminated intravascular coagulation. Compared patterns of antithrombin III, protein C, and protein S deficiencies. Chest. 1992;101:816-23.

13. Shorr AF, Bernard GR, Dhainaut JF, Russell JR, Macias WL, Nelson DR, et al. Protein C concentrations in severe sepsis: an early directional change in plasma levels predicts outcome. Crit Care Med. 2006;10:R92.

14. Faust SN, Levin M, Harrison OB, Goldin RD, Lockhart MS, Kondaveeti S, et al. Dysfunction of endothelial protein C activation in severe meningococcal sepsis. N Engl J Med. 2001;345: 408-16.

15. Hack CE. Fibrinolysis in disseminated intravascular coagulation. Semin Thromb Hemost. 2001;27:633-8. 
16. Coughlin SR. Thrombin signalling and protease-activated receptors. Nature. 2000;407:258-64.

17. Bone RC, Francis PB, Pierce AK. Intravascular coagulation associated with the adult respiratory distress syndrome. Am J Med. 1976;61:585-9.

18. Fuchs-Buder $T$, de Moerloose $P$, Ricou B, Reber G, Vifian $\mathrm{C}$, Nicod L, et al. Time course of procoagulant activity and D dimer in bronchoalveolar fluid of patients at risk for or with acute respiratory distress syndrome. Am J Respir Crit Care Med. 1996; 153:163-7.

19. Idell S, James KK, Levin EG, Schwartz BS, Manchanda N, Maunder RJ, et al. Local abnormalities in coagulation and fibrinolytic pathways predispose to alveolar fibrin deposition in the adult respiratory distress syndrome. J Clin Invest. 1989;84:695-705.

20. Idell S, Koenig KB, Fair DS, Martin TR, McLarty J, Maunder RJ. Serial abnormalities of fibrin turnover in evolving adult respiratory distress syndrome. Am J Physiol. 1991;261:L240-L8.

21. Van Till JW, Levi M, Bresser P, Schultz MJ, Gouma DJ, Boermeester MA. Early procoagulant shift in the bronchoalveolar compartment of patients with secondary peritonitis. J Infect Dis. 2006;194:1331-9.

22. Gunther A, Mosavi P, Heinemann S, Ruppert C, Muth H, Markart P, et al. Alveolar fibrin formation caused by enhanced procoagulant and depressed fibrinolytic capacities in severe pneumonia. Comparison with the acute respiratory distress syndrome. Am J Respir Crit Care Med. 2000;161:454-62.

23. Ware LB, Fang $X$, Matthay MA. Protein $C$ and thrombomodulin in human acute lung injury. Am J Physiol Lung Cell Mol Physiol. 2003;285:L514-21.

24. Matthay MA, Ware LB. Plasma protein $C$ levels in patients with acute lung injury: prognostic significance. Crit Care Med. 2004;32(5 Suppl):S229-32.

25. Bertozzi P, Astedt B, Zenzius L, Lynch K, LeMaire F, Zapol W, et al. Depressed bronchoalveolar urokinase activity in patients with adult respiratory distress syndrome. N Engl J Med. 1990;322:890-7.

26. Choi G, Wolthuis EK, Bresser P, Levi M, van der PT, Dzoljic M, et al. Mechanical ventilation with lower tidal volumes and positive end-expiratory pressure prevents alveolar coagulation in patients without lung injury. Anesthesiology. 2006;105:689-95.

27. Gray AJ, Bishop JE, Reeves JT, Mecham RP, Laurent GJ. Partially degraded fibrin(ogen) stimulates fibroblast proliferation in vitro. Am J Respir Cell Mol Biol. 1995;12:684-90.

28. Fukuda Y, Ishizaki M, Masuda Y, Kimura G, Kawanami O, Masugi Y. The role of intraalveolar fibrosis in the process of pulmonary structural remodeling in patients with diffuse alveolar damage. Am J Pathol. 1987;126:171-82.

29. Goodman RB, Pugin J, Lee JS, Matthay MA. Cytokine-mediated inflammation in acute lung injury. Cytokine Growth Factor Rev. 2003;14:523-35.

30. Pugin J, Verghese G, Widmer MC, Matthay MA. The alveolar space is the site of intense inflammatory and profibrotic reactions in the early phase of acute respiratory distress syndrome. Crit Care Med. 1999;27:304-12.

31. Park WY, Goodman RB, Steinberg KP, Ruzinski JT, Radella F, Park DR, et al. Cytokine balance in the lungs of patients with acute respiratory distress syndrome. Am J Respir Crit Care Med. 2001;164:1896-903.

32. Zapol WM, Jones R. Vascular components of ARDS. Clinical pulmonary hemodynamics and morphology. Am Rev Respir Dis. 1987;136:471-4.

33. Greene R. Pulmonary vascular obstruction in the adult respiratory distress syndrome. J Thorac Imaging. 1986;1:31-8.

34. Nuckton TJ, Alonso JA, Kallet RH, Daniel BM, Pittet JF, Eisner MD, et al. Pulmonary dead-space fraction as a risk factor for death in the acute respiratory distress syndrome. N Engl J Med. 2002;346:1281-6.

35. Idell S. Coagulation, fibrinolysis, and fibrin deposition in acute lung injury. Crit Care Med. 2003;31(4 Suppl):S213-20.

36. Rowland FN, Donovan MJ, Picciano PT, Wilner GD, Kreutzer DL. Fibrin-mediated vascular injury. Identification of fibrin peptides that mediate endothelial cell retraction. Am J Pathol. 1984;117:418-28.
37. Idell S. Extravascular coagulation and fibrin deposition in acute lung injury. New Horiz. 1994;2:566-74.

38. Marshall R, Bellingan G, Laurent $G$. The acute respiratory distress syndrome: fibrosis in the fast lane. Thorax. 1998;53:815-7.

39. Seeger W, Grube C, Gunther A. Proteolytic cleavage of fibrinogen: amplification of its surfactant inhibitory capacity. Am J Respir Cell Mol Biol. 1993;9:239-47.

40. Stevens T, García JG, Shasby DM, Bhattacharya J, Malik AB. Mechanisms regulating endothelial cell barrier function. Am J Physiol Lung Cell Mol Physiol. 2000;279:L419-22.

41. Trepat X, Grabulosa M, Buscemi L, Rico F, Farre R, Navajas D. Thrombin and histamine induce stiffening of alveolar epithelial cells. J Appl Physiol. 2005;98:1567-74.

42. Gross TJ, Simon RH, Kelly CJ, Sitrin RG. Rat alveolar epithelial cells concomitantly express plasminogen activator inhibitor1 and urokinase. Am J Physiol. 1991;260:L286-95.

43. Idell S, Kumar A, Zwieb C, Holiday D, Koenig KB, Johnson AR. Effects of TGF-beta and TNF-alpha on procoagulant and fibrinolytic pathways of human tracheal epithelial cells. Am J Physiol. 1994;267:L693-703.

44. Moalli R, Doyle JM, Tahhan HR, Hasan FM, Braman SS, Saldeen T. Fibrinolysis in critically ill patients. Am Rev Respir Dis. 1989;140:287-93.

45. Laterre PF, Wittebole $X$, Dhainaut JF. Anticoagulant therapy in acute lung injury. Crit Care Med. 2003;31(4 Suppl):S329-36.

46. Abraham E, Reinhart K, Opal S, Demeyer I, Doig C, Rodríguez AL, et al. Efficacy and safety of tifacogin (recombinant tissue factor pathway inhibitor) in severe sepsis: a randomized controlled trial. JAMA. 2003;290:238-47.

47. Warren BL, Eid A, Singer P, Pillay SS, Carl P, Novak I, et al. Caring for the critically ill patient. High-dose antithrombin III in severe sepsis: a randomized controlled trial. JAMA. 2001;286:1869-78.

48. Bajzar L, Nesheim ME, Tracy PB. The profibrinolytic effect of activated protein $\mathrm{C}$ in clots formed from plasma is TAFI-dependent. Blood. 1996;88:2093-100.

49. Macias WL, Yan SB, Williams MD, Um SL, Sandusky GE, Ballard DW, et al. New insights into the protein C pathway: potential implications for the biological activities of drotrecogin alfa (activated). Crit Care. 2005;9 Suppl 4:S38-45.

50. Nick JA, Coldren CD, Geraci MW, Poch KR, Fouty BW, O'Brien J, et al. Recombinant human activated protein $\mathrm{C}$ reduces human endotoxin-induced pulmonary inflammation via inhibition of neutrophil chemotaxis. Blood. 2004;104:3878-85.

51. Laterre PF, Garber G, Levy H, Wunderink R, Kinasewitz GT, Sollet JP, et al. Severe community-acquired pneumonia as a cause of severe sepsis: data from the PROWESS study. Crit Care Med. 2005;33:952-61.

52. Abraham E, Laterre PF, Garg R, Levy H, Talwar D, Trzaskoma BL, et al. Drotrecogin alfa (activated) for adults with severe sepsis and a low risk of death. N Engl J Med. 2005;353:1332-41.

53. Eichacker PQ, Natanson $C$. Recombinant human activated protein $\mathrm{C}$ in sepsis: inconsistent trial results, an unclear mechanism of action, and safety concerns resulted in labeling restrictions and the need for phase IV trials. Crit Care Med. 2003;31(1 Suppl):S94-6.

54. Van der Poll T, Levi M, Nick JA, Abraham E. Activated protein $\mathrm{C}$ inhibits local coagulation after intrapulmonary delivery of endotoxin in humans. Am J Respir Crit Care Med. 2005;171:1125-8.

55. Welty-Wolf KE, Carraway MS, Miller DL, Ortel TL, Ezban M, Ghio AJ, et al. Coagulation blockade prevents sepsis-induced respiratory and renal failure in baboons. Am J Respir Crit Care Med. 2001;164:1988-96.

56. Hardaway RM, Williams $\mathrm{CH}$, Marvasti M, Farias M, Tseng A, Pinon I, et al. Prevention of adult respiratory distress syndrome with plasminogen activator in pigs. Crit Care Med. 1990;18:1413-8.

57. Greene R, Lind S, Jantsch H, Wilson R, Lynch K, Jones R, et al. Pulmonary vascular obstruction in severe ARDS: angiographic alterations after i.v. fibrinolytic therapy. AJR Am J Roentgenol. 1987;148:501-8.

58. Gunther A, Lubke N, Ermert M, Schermuly RT, Weissmann N, Breithecker A, et al. Prevention of bleomycin-induced lung fibrosis by aerosolization of heparin or urokinase in rabbits. Am J Respir Crit Care Med. 2003;168:1358-65. 